\title{
Wissner, Golde / Nowack, Rebecca / Schweitzer, Friedrich / Boschki, Reinhold / Gronover, Matthias (Hg.) (2020). Jugend - Glaube - Religion II: Neue Befunde - vertiefende Analysen - didaktische Konsequenzen. Münster: Waxmann. ISBN 978-3-8309-4I55-2. 346 Seiten.
}

\author{
Alexander Unser \\ a Technische Universität Dortmund (alexander.unser@tu-dortmund.de)
}

Der zu besprechende Band ist die Fortsetzung eines im Jahr 2018 von Friedrich Schweitzer et al. herausgegebenen Buches mit dem Titel „Jugend - Glaube - Religion“, das in den RpB bereits von Matthias Werner besprochen wurde (Ausgabe 82/2020). Der hier im Fokus stehende Folgeband beschäftigt sich laut Titel mit neuen Befunden, vertiefenden Analysen und didaktischen Konsequenzen.

Es ergibt Sinn, zunächst an die den beiden Bänden zugrunde liegende Studie zu erinnern, denn vermutlich haben nicht alle Leserinnen und Leser den ersten Band von 2018 vor Augen. Die Studie steht in der Tradition der empirischen Einstellungs- und Religiositätsforschung, die in der Religionspädagogik seit mindestens zwanzig Jahren etabliert ist. So formulieren die Autorinnen und Autoren als Forschungsziel, „Einstellungen, Interessen und Fragen von Jugendlichen und jungen Erwachsenen zu Religion(en), Glaube, Kirche und dem Religions- bzw. Ethikunterricht zu erfassen“ (29). Das allein erklärt aber noch nicht die hohe (auch mediale) Aufmerksamkeit, die die Studie erfahren hat. Vielmehr ist diese Aufmerksamkeit drei Charakteristika der Studie geschuldet, die sie von bisherigen Arbeiten abhebt. Sie beansprucht, erstens, für Baden-Württemberg repräsentativ zu sein. Zweitens kombiniert sie quantitative mit qualitativen Methoden (für Band 2 weniger relevant, da dort lediglich quantitative Daten ausgewertet werden). Drittens ist die quantitative Erhebung als Panel angelegt, wodurch Veränderungen in den Einstellungen und in der Religiosität der Befragten rekonstruierbar werden.

Worum geht es aber im zu besprechenden zweiten Band? Im Kern besteht das Buch aus drei größeren thematischen Blöcken. Im ersten präsentiert Golde Wissner neue Befunde aus der dritten Erhebungswelle des Panels (43-91) sowie vertiefende Analysen zu besonderen Gruppen und Fragestellungen (95-126). Im zweiten wurden Vertreterinnen und Vertreter aus Wissenschaft und Praxis gebeten, die Befunde der Studie zu kommentieren (129-215). Im dritten greifen die Autorinnen und Autoren der Studie ausgewählte empirische Befunde auf und schließen daran religionsdidaktische Reflexionen an (219-291). Gerahmt wird dieser Kern von einer ausführlichen Einleitung, die die Befunde der Studie zusammenfasst (11-23), den Aufbau des Bandes skizziert (23-29) und noch einmal grundlegend in die Methodik der Studie einführt (29-39), sowie von einem Anhang, in dem auf vorbildliche Weise der Fragebogen, eine deskriptive Auswertung der Daten sowie die bei der Skalenkonstruktion berechneten Faktorenanalysen dokumentiert sind (295-318). Im Folgenden werden die drei thematischen Blöcke vorgestellt, wobei der Schwerpunkt auf der empirischen Studie liegen wird.

Der erste, von Golde Wissner verfasste Block bietet eine Reihe von Einzelanalysen, die hier aufgrund ihrer Fülle nicht umfassend dargestellt werden können. Es lohnt sich aber, Zeit für die Lektüre zu investieren, da sich interessante Einblicke in die Glaubensentwicklung Jugendlicher bzw. junger Erwachsener gewinnen lassen. Zunächst dokumentiert die Autorin, wie sich die Einstellungen der Befragten zu verschiedenen religiösen Inhaltsbereichen im Zeitraum von drei Jahren entwickelt haben $(\mathrm{t} 1 \rightarrow \mathrm{t}$ ). Insgesamt liegen für $\mathrm{N}=599$ Befragte Daten zu allen drei Messzeitpunkten vor. Neben Einstellungen zu Glaube und Religion (45-54), einem Leben nach dem Tod (55-58), verschiedenen Gottesvorstellungen (58-64), der Vereinbarkeit von Glauben und Naturwissenschaft (64- 66) sowie zur Kirche (66-72), werden auch das Interesse an Glaubensfragen (72-75), Einstellungen gegenüber anderen Religionen (76-79) sowie die (rückblickende) Bewertung des Religions- bzw. Ethikunterrichts thematisiert (79-87). Viele der Einstellungen bleiben über die Zeit konstant. In manchen Bereichen lassen sich aber deutliche Einstellungsänderungen wahrnehmen, von denen hier zumindest vier genannt werden sollen. So lässt sich eine deutliche Zunahme a-personaler Gottesvorstellungen (deistisch, pantheistisch) bei den Befragten beobachten, die jedoch stark mit der Vorstellung verknüpft sind, dass man zu Gott sprechen kann $(r=.508)(58-61)$. Weiter zeichnet sich eine zuneh- 
mende kritische Haltung zu und Distanzierung von Kirche als religiöser Institution ab (67-68). Dagegen nimmt die Auseinandersetzung mit Glaubens- und Sinnfragen zu (73-75) und Religions- bzw. Ethikunterricht werden retrospektiv positiver bewertet hinsichtlich ihres Beitrags, Denkanstöße gegeben zu haben und bei schwierigen Lebensfragen zu helfen (80-81).

Weiter nimmt die Autorin vertiefende Analysen zu besonderen Gruppen und Fragestellungen vor (95- 126). Diese Analysen beziehen sich nun aber nicht mehr auf Einstellungsänderungen von $\mathrm{t} 1$ nach $\mathrm{t} 3$, sondern stellen eine Momentaufnahme des ersten Erhebungszeitpunktes t1 mit dem entsprechend größeren Sample von N=7.246 Befragten dar. Die Auswahl der Gruppen und Themen ist motiviert durch „Diskussionen mit Expertinnen und Experten aus Praxis und Wissenschaft" (95). So werden Jugendliche ohne Religionszugehörigkeit näher analysiert (96-104) und es wird untersucht, welche unterschiedlichen Bedeutungen die Befragten den Begriffen „gläubig“ und "religiös“ beimessen (104-110), wie sich der Glaube von Religions- und Ethikunterrichtsschülerinnen und -schülern unterscheidet (110-116), ob es antisemitische und islamophobe Einstellungen bei den muslimischen Befragten gibt (116-121) und welche Bedeutung die Theodizeefrage für die Befragten hat (121-126).

Im zweiten thematischen Block des Bandes kommen eine Reihe von Expertinnen und Experten zu Wort, die die Studienergebnisse kommentieren. Aus wissenschaftlicher Sicht reflektiert Ulrich Kropač die Bedeutung ungebundener Religiosität für religiöse Bildungsprozesse (129-137), Fahimah Ulfat Veränderungen in der Religiosität von Musliminnen und Muslimen (138-144) und Elisabeth Naurath die Bedeutung ethischer Bildung im Religionsunterricht (145-153). Ulrich Riegel (154-161) und Werner Helsper (162-174) bewerten jeweils das methodische Design der Studie. Konsequenzen für die schulische Praxis werden schließlich von Bernhard Riesch-Clausecker (175-184), Uta Martina Hauf (185-194), Peter Kliemann (195-204) und Christof Schilling (205-215) formuliert. Gerade bei letzteren Beiträgen ist es interessant zu sehen, welche Studienergebnisse von Praxisakteurinnen und -akteuren wie rezipiert und in den eigenen Erfahrungskontext integriert werden.

Schließlich legen die Autorinnen und Autoren der Studie im dritten thematischen Block didaktische Analysen zu ausgewählten empirischen Befunden vor (219-291). Der Aufbau der Kapitel ist parallel gestaltet und orientiert sich am Dreierschema Sehen-Urteilen-Handeln. So werden, erstens, nochmals zentrale Studienergebnisse referiert, um diese, zweitens, didaktisch zu bewerten und, drittens, Handlungskonsequenzen zu skizzieren. Auch hier wird eine Fülle von Themen bearbeitet, die das Verhältnis von Religion und Glaube (219-228), Wahrnehmungen des Religions- und Ethikunterrichts (229-236), Jenseitsvorstellungen (237-244), Glaube und Naturwissenschaft (245-254), Gottesbilder (255-263), Kritik an der Kirche (264-270), Einstellungen gegenüber anderen Religionen (271-278), Theodizee (279-284) und die persönliche Relevanz von Religion (285-291) betreffen.

Insgesamt lässt sich sagen, dass der vorliegende Band eine Fülle von Themen und Perspektiven abdeckt, die es verschiedenen Leserinnen und Lesern ermöglicht, von den Studienergebnissen zu profitieren. Dass die Studie so über verschiedene Wege zugänglich gemacht wird, ist eine der großen Stärken des Bandes. Bei 346 Seiten bleibt bei dieser Fülle dann aber auch nur beschränkt Platz für Einzelnes. Ich hätte mir beispielsweise im ersten Themenblock gewünscht, dass neben den Beschreibungen der Einstellungsänderungen auch mehr Analysen vorgenommen werden, die diese Veränderungen erklären. Daher bleibt zu hoffen, dass dieser interessante Band Ausgangspunkt für eine Reihe weiterer Publikationen ist, die die gewonnenen Einsichten weiter vertiefen. 